\subsection{8.-03.09.2011}

Hamburg

Deutschland

\subsection{9.-14.09.2011 \\ Los Angeles, CA}

USA

\section{[............... \\ 14.09.-17.09.2011 \\ Leipzig}

Deutschland
XVI. World Congress of the International Federation for the Surgery of Obesity and Metabolic Disorders
Auskunft: INTERPLAN Congress,

Meeting \& Event Management AG

Landsberger Straße 155

80687 München

Tel. +49 89 548234-0, Fax -44

info@interplan.de

www.ifso2011.de

Auskunft: $\mathrm{CPO}$ Hanser Service $\mathrm{GmbH}$

Paulsborner Straße 44

14193 Berlin

Tel. +49 30 30-06690, Fax -57391

ice2011@cpo-hanser.de

www.ice2011.org

\section{Viszeralmedizin 2011}

Auskunft: INTERPLAN Congress,

Meeting \& Event Management AG

Landsberger Straße 155

80687 München

Tel. +49 89 548234-0, Fax -44

viszeralmedizin@interplan.de

www.viszeralmedizin.com

\section{Erlangen}

Deutschland

\section{…...........}

Konstanz

Deutschland

\subsection{9.-17.09.2011}

Innsbruck

Österreich

...............

23.09.2011

Linz

Österreich

..................

05.10.-08.10.2011

Innsbruck

Österreich

\subsection{0.-08.10.2011}

St. Wolfgang

Österreich

.................

08.10 .2011

Erlangen

Deutschland

..................

13.10.-16.10.2011

Stuttgart

Deutschland
27. Jahreskongress der Deutschen Gesellschaft für Gefäßchirurgie und Gefäßmedizin

Auskunft: INTERPLAN Congress,

Meeting \& Event Management AG

Landsberger Straße 155

80687 München

Tel. +49 89 548234-795, Fax -44

dgg2011@interplan.de

www.dgg-jahreskongress.de

48. Kongress der Südwestdeutschen Gesellschaft für Innere Medizin

Auskunft: MEDICA Deutsche Gesellschaft

für Interdisziplinäre Medizin e.V.

gw@medica-ev.de

www.medcongress.de

Blutungskurs (ÖGGH)

Auskunft: azmedinfo@media.co.at

www.oeggh.at

Risikomanagement in der Endoskopie

42. Jahrestagung der Österreichischen Gesellschaft für Innere Medizin (ÖGIM)

Auskunft: azmedinfo@media.co.at www.oeggh.at

Auskunft: pco tyrol congress

Rennweg 3

6020 Innsbruck

Tel. +43512 5756-00, Fax -07

oegim2011@come-innsbruck.at

www.oegim.at

Auskunft: www.medacad.org/senologie-aco2011

Gemeinsame Jahrestagung ACO-ASSO und Senologie -

Mammakarzinom, State-of-the-Art

Auskunft: www.drfalkpharma.de

Endoskopieseminare Stuttgart 2011
Update Gastroenterologie \& Hepatologie 2011
Auskunft: Klinikum Stuttgart - Katharinenhospital Frau E. Kassnel

Tel. +49711 27835401

e.kassnel@klinikum-stuttgart.de

www.klinikum-stuttgart.de

\section{KARGER}

Fax +497614520714

Information@Karger.de

www.karger.com (c) 2011 S. Karger GmbH, Freiburg 


14.10.-15.10.2011
Graz
Österreich
$\ldots \ldots \ldots \ldots \ldots$
$14.10 .-15.10 .2011$
Innsbruck
Österreich
$\ldots \ldots \ldots \ldots$
$20.10 .-21.10 .2011$
Bochum
Deutschland
@................
$22.10 .-26.10 .2011$
Stockholm
Schweden

EASIE-ERCP-Kurs (ÖGGH)

Mukosektomie/Polypektomie-Kurs (ÖGGH)

178. Jahrestagung Vereinigung Niederrheinisch-Westfälischer Chirurgen

19th United European Gastroenterology Week

Schweden

29.10.2011
Wien
Österreich
$\cdots \cdots \ldots \ldots \ldots \ldots \ldots$
04.11.-05.11.2011
Hamburg

Deutschland

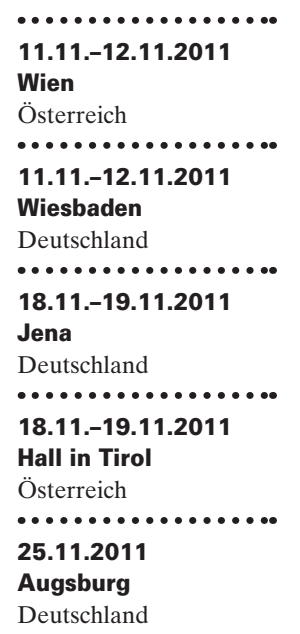

[.................

\subsection{1.-26.11.2011}

Krefeld

Deutschland

[...................

25.11.-26.11.2011

Wien

Österreich

[.................

01.12.-03.12.2011

Hamburg

Deutschland

\section{$02.12 .-03.12 .2011$}

Linz

Österreich

[..................

20.01.-21.01.2012

München

Deutschland

\section{Sedoanalgesie-Kurs (ÖGGH)}

\section{Endo Club Nord}

Endoskopie Postgraduiertenkurs (ÖGGH)

1. Endoskopie-Sonografie-Update

8. Symposium der Arbeitsgemeinschaft Kinderurologie der Deutschen Gesellschaft für Kinderchirurgie

Update Gastroenterologie 2011

Endo-Update 2011

186. Tagung der Rheinisch-Westfälischen Gesellschaft für Innere Medizin

\section{Endoskopiekurs für Anfänger (ÖGGH)}

188. Tagung der Vereinigung Nordwestdeutscher Chirurgen

\section{EASIE EMR und ESD (ÖGGH)}

10. Gastro Forum München
Auskunft: azmedinfo@media.co.at www.oeggh.at

Auskunft: azmedinfo@media.co.at www.oeggh.at

Auskunft: www.nrwchirurgen.de

Auskunft: CPO Hanser Service $\mathrm{GmbH}$

Paulsborner Straße 44

14193 Berlin

Tel. +49 30 30-06690, Fax -57391

uegw2011@cpo-hanser.de

www.uegf.org

Auskunft: azmedinfo@media.co.at

www.oeggh.at

Auskunft: $\mathrm{COCS} \mathrm{GmbH}$

Congress Organisation C. Schäfer

Franz-Joseph-Straße 38

80801 München

Tel. +49 89 30710-11, Fax -21

www.cocs.de

Auskunft: azmedinfo@media.co.at

www.oeggh.at

Auskunft: kathrin.schoppa@ed-update.com www.endo-sono-update.com

Auskunft: ag-kinderurologie@dgkch.de

www.kinderchirurgie.uniklinikum-jena.de

Auskunft: azmedinfo@media.co.at

Auskunft: Sekretariat III. Medizinische Klinik,

Klinikum Augsburg

Frau Konzet

gudrun.konzet@klinikum-augsburg.de

www.klinikum-augsburg.de

Auskunft: www.rwgim.de

Auskunft: azmedinfo@media.co.at

www.oeggh.at

Auskunft: $\mathrm{MCN}$ Medizinische Congressorganisation Nürnberg AG

Frau Reimer

Tel. +49 911 39316-40, Fax -66

reimer@mcn-nuernberg.de

www.mcn-nuernberg.de

Auskunft: azmedinfo@media.co.at

www.oeggh.at

Auskunft: $\mathrm{COCS} \mathrm{GmbH}$

Congress Organisation C. Schäfer

Tel. +49 89 890677-0, Fax -77

Frau Reber

sandra.reber@cocs.de

www.cocs.de

Tagungen und Kongresse

Meetings and Conferences 
02.2012

Düsseldorf

Deutschland

\section{.................}

22.02.-25.02.2012

Berlin

Deutschland

...................

25.02.2012

Ludwigshafen

Deutschland

\subsection{3 .04 .03 .2012}

Nürnberg

Deutschland

\section{[................}

22.03.-24.03.2012

München

Deutschland

[................

22.03.-24.03.2012

St. Gallen

Schweiz

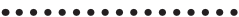

24.04.-27.04.2012

Berlin

Deutschland

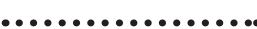

27.09.-29.09.2012

Karlsruhe

Deutschland
14. Internationales Endoskopie Symposium Düsseldorf

30. Deutscher Krebskongress

VII. "LUKS" - Ludwigshafener und Kölner Stentworkshop

Gemeinsamer Bundeskongress Chirurgie 2012

42. Kongress der Deutschen Gesellschaft für Endoskopie und Bildgebende Verfahren e.V.

\section{1st St. Gallen EORTC Gastrointestinal Cancer Conference}

(SG-GICC 2012)

129. Kongress der Deutschen Gesellschaft für Chirurgie (DGCH)

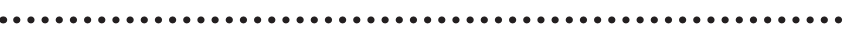

21. Jahrestagung der Deutschen Gesellschaft für Thoraxchirurgie (DGT)
Auskunft: $\mathrm{COCS} \mathrm{GmbH}$ Congress Organisation

C. Schäfer

Frau Reber

sandra.reber@cocs.de

www.endo-duesseldorf.com

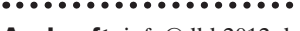

Auskunft: info@dkk2012.de

$\ldots \ldots \ldots \ldots \ldots \ldots \ldots \ldots \ldots \ldots \ldots \ldots \ldots \ldots \ldots \ldots \ldots$

Auskunft: $\mathrm{COCS} \mathrm{GmbH}$ Congress Organisation

C. Schäfer

Frau Gautsch

eva.gautsch@cocs.de

www.cocs.de

0

Auskunft: MCN Medizinische Congressorganisation

Nürnberg AG

Tel. +49 911 39316-39, Fax -20

bnc@mcnag.info

www.mcn-nuernberg.de

Auskunft: $\mathrm{COCS} \mathrm{GmbH}$ Congress Organisation

C. Schäfer

Frau Wiederkrantz

Tel. +49 89 890677-0, Fax -77

martina.wiederkrantz@cocs.de

www.dge-bv.de

•..................

Auskunft: St. Gallen Oncology Conferences

c/o Tumor and Breast Center ZeTuP

info@oncoconferences.ch

www.oncoconferences.ch

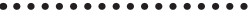

Auskunft: MCN Medizinische Congressorganisation Nürnberg AG

Frau Veith

veith@mcn-nuernberg.de

www.chirurgie2012.de

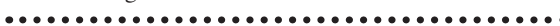

Auskunft: MCN Medizinische Congressorganisation

Nürnberg AG

mcn@mcn-nuernberg.de

www.dgt-online.de 